\title{
Relacionamiento Entre los Talleres de Arquitectura y el Laboratorio de Fabricación Digital
}

\author{
Relationship Between Academic Architectural Workshops and Digital Fabrication Laboratory
}

\author{
Arq. Paulo Adhemar Pereyra Bonifácio \\ Facultad de Arquitectura, Diseño y Urbanismo \\ Universidad de la República, Uruguay \\ paulopereyra@fadu.edu.uy \\ Bach. Ximena Echavarría Terradas \\ Facultad de Arquitectura, Diseño y Urbanismo \\ Universidad de la República, Uruguay \\ pimets@fadu.edu.uy
}

\author{
Bach. Luis Flores \\ Facultad de Arquitectura, Diseño y Urbanismo \\ Universidad de la República, Uruguay \\ Iflores@fadu.edu.uy \\ Bach. Lucía Meirelles \\ Facultad de Arquitectura, Diseño y Urbanismo \\ Universidad de la República, Uruguay \\ kuchiki@fadu.edu.uy
}

\begin{abstract}
This article develops the relationship between academic architectural workshops with digital fabrication laboratory. We understand that it is essential to implement informatics to design, especially the case of digital manufacturing, as an active tool in the design process and project representation.
\end{abstract}

Keywords: Arquitectura; Fabricación Digital; Anteproyecto Proyecto.

\section{Introducción}

El presente artículo aborda el marco de relacionamiento entre los talleres de Anteproyecto y Proyecto de Facultad de Arquitectura con el laboratorio de fabricación digital.

El planteo de su estudio responde:

- A la gran demanda que aumenta de forma exponencial de solicitudes de trabajos con las tecnologías que disponemos en el laboratorio. Teniendo en cuenta la acotada disponibilidad de recursos humanos y de equipamiento. Esto nos ha generado un gran desafío ya que llegamos al punto de saturación, en donde las labores de investigación propiamente del laboratorio, quedan desplazadas para atender dicha demanda. Esto sucede durante algunos períodos específicos, principalmente sobre finales de junio y de noviembre, en donde los talleres de anteproyecto realizan sus entregas semestrales. La demanda por parte de estudiantes proviene primeramente de la carrera de Arquitectura, pero también de la Escuela de Diseño Industrial. En ambas carreras son varias las materias que requieren el prototpipado y/o la representación en forma de maqueta del proyecto.

- La posibilidad que generan dichas instancias de relacionamiento, con el fin de realizar procesos de transferencia tecnológica de los principios rectores de las distintas tecnologías de Fabricación Digital como son las sustractivas, aditivas, conformadas e híbridas.

Los objetivos que se persiguen, considerando ese aumento significativo de la demanda y esa posibilidad de transferencia, son los siguientes:

1. Consolidar el desarrollo del Laboratorio de Fabricación Digital como espacio integrador de tecnologías, técnicas, software, hardware y materiales, atendiendo aspectos de gestión específicos tales como: uso, difusión, mantenimiento, seguridad e higiene.

2. Explicitar los principios rectores de las tecnologías de fabricación digital, promoviendo la creación, profundización y divulgación de conocimientos y saberes relativos a la disciplina de fabricación digital enfocada en resolver problemas de diseño en general y de la producción arquitectónica en particular. 


\section{Procedimientos Metodológicos}

\section{Aproximación al problema}

El actual Plan de Estudios mantiene la figura del Taller de Anteproyecto y Proyecto como ámbito privilegiado de reflexión y aprendizaje de la Arquitectura, asignándole un rol estructurador en la formación del arquitecto "El Área Proyectual debe formar al estudiante en todas las escalas: del edificio, urbana y territorial. El estudiante adquirirá conocimientos, capacidades y herramientas para las distintas actividades involucradas, como ser: análisis, programación, diseño y evaluación. El Área Proyectual deberá integrar y poner en valor los conocimientos aportados a la actividad proyectual desde las otras áreas, tanto en los cursos de Anteproyecto como en el de Proyecto." (Plan de Estudios, 2002, p. 7) En efecto, es el momento en que el diseño y la composición arquitectónica se ven enriquecidos por la integración de los saberes específicos de otras disciplinas. Sin embargo, el nuevo Plan no integra en la formación curricular los conocimientos de las disciplinas emergentes TIC en general y de la Informática Aplicada (cad/cam) de las tecnologías de Fabricación Digital en particular.

Por otra parte, hay una instancia fundamental dentro del desarrollo de los Talleres que es la entrega final. Los cursos de Anteproyecto se dictan en forma semestral, teniendo una instancia de presentación de los proyectos. Tradicionalmente se presenta una lámina y el complemento de una maqueta, en representación del proyecto presentado.

En función de ello, los Talleres, así como los estudiantes, solicitan trabajos al labFab_MVD, que incluyen algunas de las funciones y tecnologías que pasamos a detallar:

- $\quad$ Procesamiento de corte, grabado y tallado de curvas de niveles altimétricas aptas para ser utilizadas como soporte urbano/territoriales en las que se insertan las volumetrías arquitectónicas.

- $\quad$ Generación de "artefactos" para su posterior armado, a modo de puzzles tridimensionales con distintas técnicas.

- Elaboración de objetos como apoyo a las instalaciones de entrega.

- $\quad$ Cortes múltiples de placas/soporte de entregas con estampado de logos.

- Cortes múltiples para armado de maquetas con tecnologías sustractivas.

- Impresión 3d de maquetas arquitectónicas.
Mediante la reflexión sobre los antecedentes previamente mencionados, y entendiendo la problemática existente, es que se planifican una serie de cursos, exposiciones y actividades con el fin de estimular y promocionar la integración de la fabricación digital en el proceso de diseño del proyecto arquitectónico.

Dado que la mayoría de los estudiantes de nuestra facultad no están familiarizados con el diseño con fabricación digital, es necesaria la implementación de una instancia de enseñanza/aprendizaje de las tecnologías de fabricación. A partir de la consideración del hecho de que la Informática Aplicada (IA) no fue incluida como disciplina en el nuevo Plan de Estudios, el Departamento de Informática Aplicada al Diseño se empeña en incluir módulos con contenidos de IA en las unidades curriculares, que puedan tener relación, de todas las carreras de grado y posgrado de la Facultad.

El problema principal que nos planteamos es como integrar los tradicionales medios con los contemporáneos, como son la prefiguración, modelización y materialización, al proceso de diseño, no tanto como poderosos recursos instrumentales, sino como un nuevo paradigma para la apropiación de estrategias que supongan un cambio cualitativo en las formas de componer y proyectar. En ese sentido, y con la finalidad de explicitar los conocimientos específicos de las tecnologías de fabricación digital se dicta de forma semestral la materia opcional Fabricación con Diseño Digital. Dicha materia está orientada a estudiantes de todas las carreras que se dictan en la Facultad, perteneciente al segundo ciclo, por lo que son estudiantes avanzados, y en muchos casos, finalizando la carrera. Las materias opcionales buscan generar una formación en áreas específicas, elegidas de forma individual, por el estudiante.

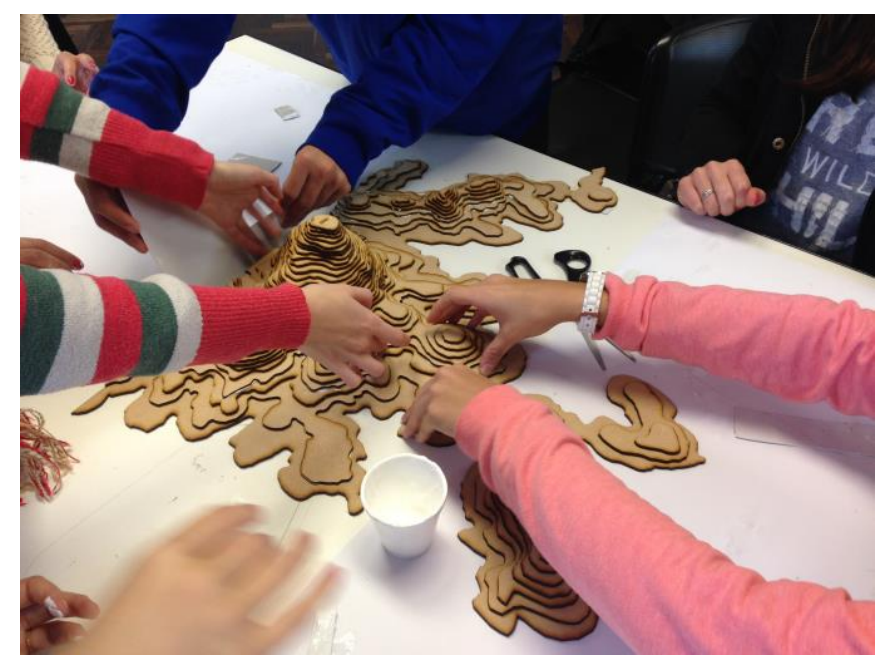

Figura 1: estudiantes en el armado de maqueta de terreno Taller Berio 
El objetivo es incluir en el proceso y diseño con fabricación digital al estudiante en todo el transcurso de su carrera, desde sus primeros años. Por este motivo se está planificando un curso de Introducción a las tecnologías de fabricación digital, que a modo de propedéutico, posibilite explicitar las posibilidades sobre las condicionantes necesarias para diseñar y fabricar con las tecnologías disponibles. Es fundamental tener en claro cómo funcionan, y los elementos necesarios para llevar a cabo la fabricación del prototipo y/o maqueta de proyecto. Por esta razón, se incluye en el curso, un desarrollo sobre el mecanizado y proceso de cada una de las tecnologías. A esto se complementa una serie de protocolos de gestión, uso, mantenimiento, seguridad e higiene y publicación. Dichos protocolos pretenden optimizar el flujo de trabajo así como transmitir y difundir los procesos de materialización llevados a cabo en el labFab_MVD.

\section{Funcionamiento del labFab_MVD}

El laboratorio es un espacio de investigación abierto a todos los estudiantes y docentes de todas las carreras de la FADU, por lo que afecta de forma transversal al estudiante. El objetivo es que el estudiante y/o docente se acerque al laboratorio para llevar a cabo un proceso de investigación sobre su proyecto o prototipo con el apoyo de los docentes del labfab. Este proceso se da desde los primeros años de las carreras hasta los últimos. Pudiendo acceder y aprender las diferentes tecnologías, y lo que es más importante, el proceso de diseño y dibujo que hay detrás de cada una de ellas, considerando la multiplicidad de factores y herramientas disponibles.

Con el fin de promover el espacio de investigación, el primer semestre del presente año, se puso en funcionamiento el Seguimiento de proyecto. Esta propuesta se basa en el interés por parte del estudiante, de representar o prototipar su proyecto. Con la tutoría de uno de los docentes del laboratorio, quien será de referencia durante todo el semestre, se comienza una etapa de investigación con las diferentes tecnologías acorde al proyecto. En este proceso se pretende llevar a cabo una transferencia tanto técnica como tecnológica, para lograr que la fabricación no sea un mero aporte a un proyecto, sino que logre ser una herramienta de investigación del proyecto y esté disponible desde la etapa del diseño hasta la representación final del mismo.

Al mismo tiempo se implementó un sistema de agenda, con el fin de evitar la saturación, tanto del local como de los recursos. Lo principal de la agenda es la previa revisión de archivos y proyecto, con especial énfasis en aquellos que no se encuentran dentro del Seguimiento de proyecto. Esto genera una instancia previa en la cual el docente puede comprender el proyecto, y por consiguiente, su forma de fabricarlo. En general es necesario un ajuste sobre los dibujos y/o modelados, ya sean para tecnologías aditivas como sustractivas. Un vez realizado el ajuste, se agenda día y hora para llevar adelante el proceso.

Conjuntamente con el protocolo perteneciente a cada una de las tecnologías, se encuentran: el Protocolo General de Uso del labFab, el protocolo de Seguridad e Higiene, y se adicionó el Formulario de Autorización de Uso de Imágenes. Éste último resulta fundamental para llevar a cabo un registro fotográfico de todo lo que se fabrica en el laboratorio. Al mismo tiempo colabora en la difusión del labFab, ya que es necesaria la firma del docente a cargo del curso para el cual está realizando la investigación.

\section{Estudio de casos}

Anteproyecto II - Taller Schelotto

Anteproyecto II es una materia que pertenece al tercer año de la carrera de Arquitectura. La propuesta para este proyecto por parte del taller fue trabajar en dos predios distintos, ubicados en las zonas de Colonia y Carmelo respectivamente. La escala de los proyectos es media, afectando a un predio específico de su entorno.

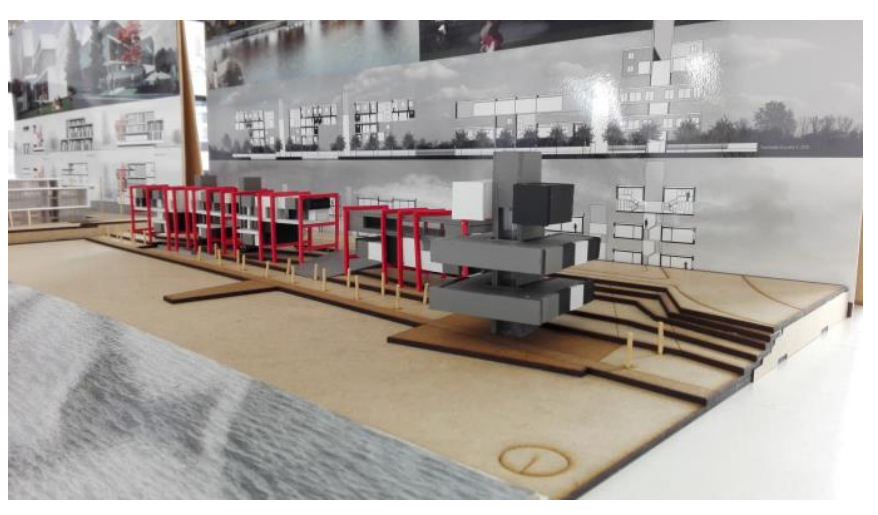

Figura 2: maqueta de estudiante - Taller Schelotto

El intercambio con el laboratorio surgió por parte de los docentes con el fin de fabricar el terreno como base de estudio del proyecto. Cada estudiante proyectaría su propuesta sobre el terreno fabricado, modificándolo y realizando los ajustes pertinentes a cada proyecto, para luego fabricar cada terreno de forma individual. 


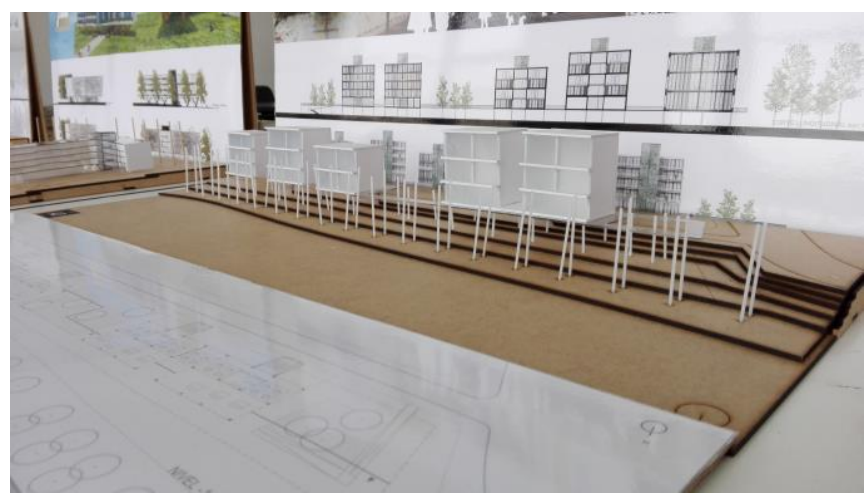

Figura 3: maqueta de estudiante - Taller Schelotto

El proceso comenzó por la corrección del diseño del terreno para darle a éste una lógica con fabricación digital. Esto facilitará su armado logrando la suficientemente firmeza, para colocar sobre éste la maqueta del proyecto, evitando desperdicios innecesarios de material. Una vez diseñado el terreno se realizaron dos pruebas, una de cada terreno, como verificación del diseño. Estas maquetas de prueba se tomaron como referencia, dejando las maquetas de los estudiantes para finales de semestre, ya que en su mayoría se plantearon variaciones en el terreno.

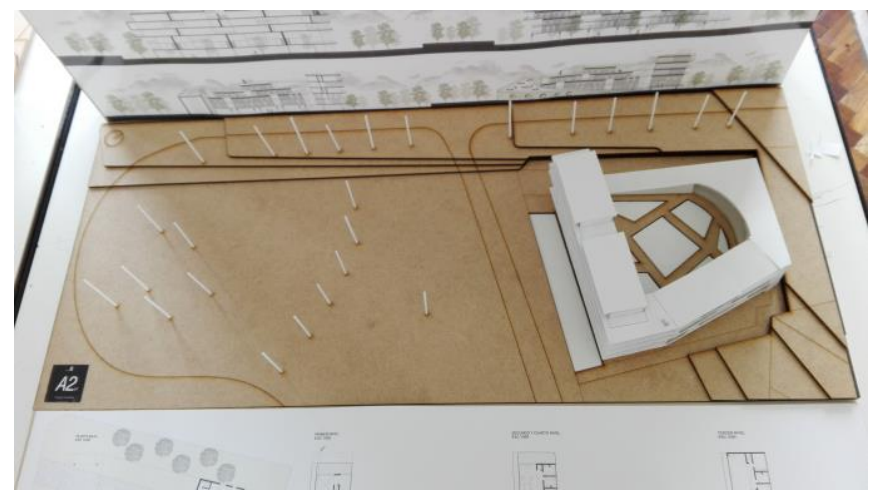

Figura 4: maqueta de estudiante - Taller Schelotto

Sobre el final del primer semestre, tuvimos una instancia con cada estudiante, con el fin de realizar las correcciones pertinentes del archivo para corte láser, al mismo tiempo que se les explicaba el funcionamiento de la tecnología, y los cuidados que se deben tener en cuanto al diseño para la fabricación digital.

La etapa de correcciones individuales junto a los cortes de las maquetas tomaron 5 jornadas de trabajo de aproximadamente 4 horas por jornada.

Proyecto Final - Taller Articardi

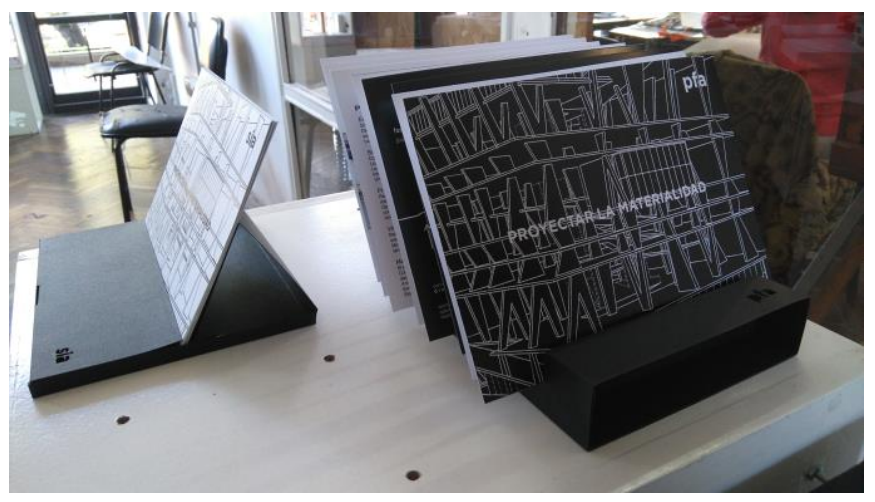

Figura 5: cajas expositoras - Taller Articardi

En este caso los docentes de Carpeta, materia que pertenece al último año de la carrera acorde al Plan de Estudios vigente, solicitaron hacer uso de la tecnología disponible en el laboratorio para armar la exposición de las entregas. Su exposición contaría con una serie de cajas plegadas a modo de expositores, y unas placas de mdf a modo de indicadores de proyectos.

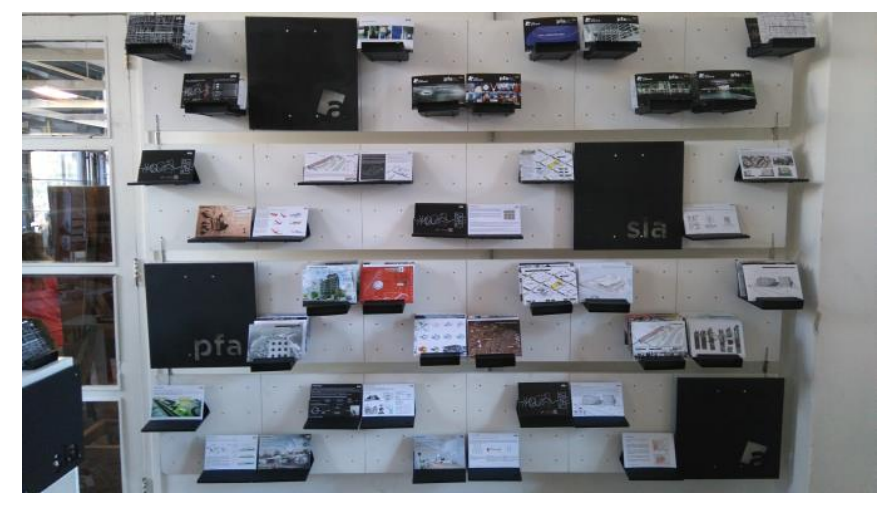

Figura 6: cajas expositoras - Taller Articardi

Las cajas plegadas serían de papel color negro con un gramaje de $270 \mathrm{~g} / \mathrm{m}^{2}$. Para realizar estos cortes se realizaron diferentes pruebas en la Cortadora Láser, con el objetivo de lograr la mejor eficiencia de la tecnología con el menor quemado posible. Para los cortes en mdf, se utilizó el Router CNC debido a su espesor.

\section{Proyecto Final - Taller Danza}

En el caso del Taller Danza, se requerían soportes verticales en mdf, procesando un total de 34 placas con la tecnología con Router CNC. Sobre éstos paneles serán colgados los ploteos de final de carrera. Se prevén las perforaciones para su posterior colgado y se incluye el logo del taller. 


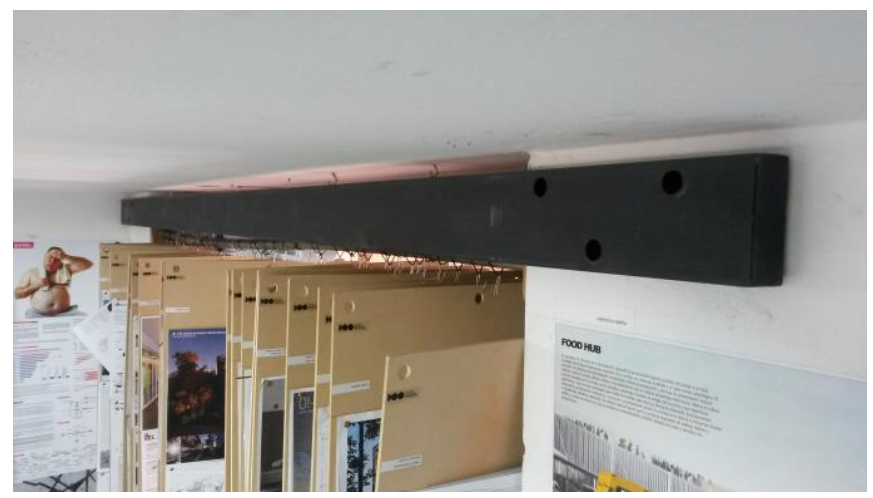

Figura 7: placas expositoras - Taller Danza

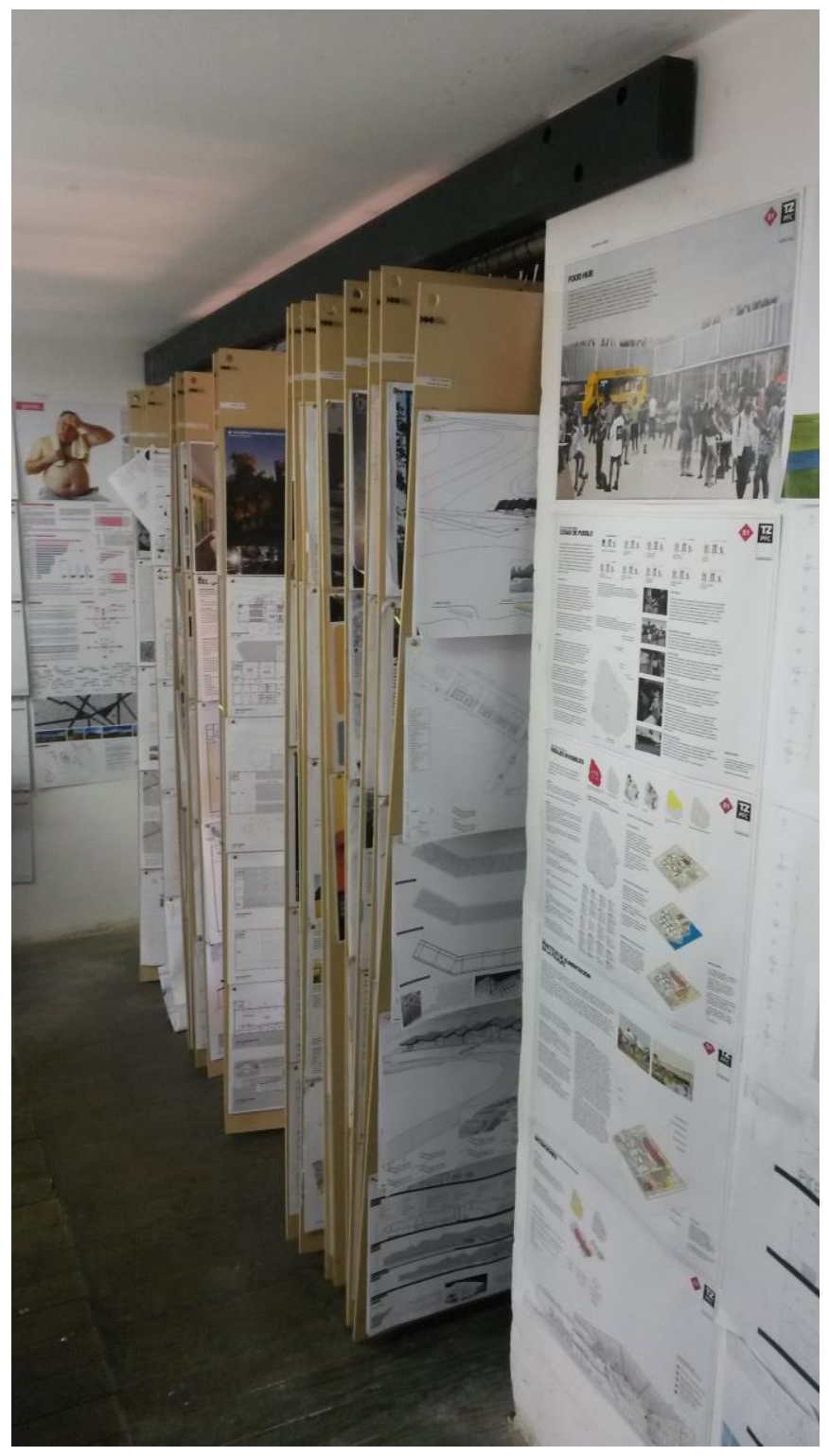

Figura 8: placas expositoras - Taller Danza

\section{Resultados Esperados}

Comenzamos en el presente año, a implementar los sistemas de agenda, formularios de autorización, Seguimiento de proyecto y los diferentes protocolos como ya detallaron anteriormente. Con estos lineamientos y estrategias de trabajo, se esperan obtener los siguientes resultados:

- Incentivar a los talleres a incluir el uso de las tecnologías de fabricación digital como parte del proceso de la investigación proyectual Esto genera una retroalimentación constante entre los Talleres y el laboratorio, y enriquece el proceso proyectual.

- Lograr la máxima difusión del conocimiento de las tecnologías y su metodología de diseño y fabricación mediante el curso Introducción a las tecnologías de fabricación digital.

- Optimización de los tiempos gracias a la organización propuesta haciendo de los tiempos más productivos tanto como para los talleres (estudiantes y docentes) como para el área de investigación del Laboratorio.

\section{Discusión}

Tras la investigación de los trabajos en forma conjunta con los Talleres de Anteproyecto podemos concluir que se cumplen los resultados esperados. Se lograron optimizar los tiempos generales, gracias a la organización por agenda previa verificación del archivo, y una mejor organización por la implementación de los protocolos. Hubo una mayor difusión por parte de los docentes de las diferentes materias, siendo en su mayoría docentes de Anteproyecto. Y se comprobó lo positivo y colaborativo del trabajo en forma conjunta con los talleres, ya que cada estudiante y/o docente representa una nueva instancia de investigación. $Y$ la fabricación digital resulta una excelente vía de representación de los proyectos dada la multiplicidad de tecnologías disponibles.

Esto genera una posibilidad de representación gráfica contemporánea, incluyendo los procesos de prefiguración, modelado y materialización, todos integrados en el proceso de diseño que se plantea como un nuevo paradigma para la apropiación de estrategias que suponen un cambio cualitativo en las formas de componer y proyectar.

\section{Agradecimientos}

Agradecemos a los Talleres de Anteproyecto y Proyecto Schelotto, Articardi, Berio y Danza por el apoyo para llevar a cabo esta investigación. 


\section{Referencias}

Pupo, R., \& Celani, M. G. C. (2011). Prototipagem rápida e fabricação digital na Arquitetura: fundamentação e formação. In: Kowaltowski, D. C. C. K., Moreira, D. de C.,Petreche, J.R. D., \& Fabrício, M . M. (Org.). O Processo de Projeto em Arquitetura da Teoria à Tecnologia. São Paulo: Oficina de Textos.

Kolarevic, Branko. Architecture in the Digital Age Design and Manufacturing, 2003, Spon Press, New York and London.

Gershenfeld, Neil; (2005) Fab. The Coming Revolution on Your Desktop - From Personal Computers to Personal Fabricaction. Nueva York: Basic Books.

Schodek, Daniel; Bechthold, Martin; Griggs, James Kimo. (2004). Digital Design and Manufacturing: CAD/CAM Applications in Architecture and Design. New Jersey: John Wiley \& Sons, Hoboken.

Iwamotto, Lisa; Digital Fabrications. (2009) Architectural and Material Techniques. Nueva York: Princeton Architectural Press.

Oxman, Rivka. (2008). Performance-based Design: Current Practices and Research Issues. International Journal of Architectural Computing, 6, 1-17. 2016, De International Journal of Architectural Computing Base de datos.

Consejo de Facultad de Arquitectura UDELAR. (2002). Plan de estudios 2002. 2016, de UDELAR Sitio web: http://www.farq.edu.uy/arquitectura/files/2011/10/plan_de_est udios_2002-1.pdf

Consejo de Facultad de Arquitectura UDELAR. (1952). Plan de estudios 1952. 2016, de UDELAR Sitio web: http://www.farq.edu.uy/bedelia/files/2012/03/Plan-de-

Estudios-1952-Anual.pdf 